\title{
Influence of Mode of Delivery on the Stress Response in Infants
}

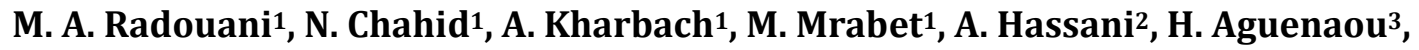 \\ Amina Barkat ${ }^{1,4}$ \\ ${ }^{1}$ Research Team on Health and Nutrition of Mother and Child, Faculty of Medicine and Pharmacy of Rabat, \\ Mohammed V University of Rabat, Rabat, Morocco \\ ${ }^{2}$ University of Abulcassiss, Rabat, Morocco \\ ${ }^{3}$ Mixed Research Unit in Nutrition and Food URAC 39, (University Ibn Tufail-CNESTEN) Designated Regional \\ Center of Nutrition Associate AFRA/IAEA, Kénitra, Morocco \\ ${ }^{4}$ Department of Medicine and Neonatal Resuscitation, National Reference Center for Neonatology and \\ Nutrition, Children's Hospital, Ibn Sina Hospital, Souissi, Morocco \\ Email: "barakatamina@hotmail.fr
}

Received 31 July 2015; accepted 10 November 2015; published 13 November 2015

Copyright (C) 2015 by authors and Scientific Research Publishing Inc.

This work is licensed under the Creative Commons Attribution International License (CC BY). http://creativecommons.org/licenses/by/4.0/

(c) (i) Open Access

\begin{abstract}
Objective: Analysis of stress response of newborns born vaginally assisted compared to those born by caesarean section scheduled at birth and then 15 days to 2 months of life. The methodology is based on: 1) The neonatal period, the analysis of cortisol levels in the blood of the umbilical artery by Radio-Immuno-Assay, 2) At the age of 15 days and two months, evaluation of the intensity of the pain response to vaccine act using DAN scores. The results of the first part of the study showed that both groups were comparable regarding maternal and neonatal characteristics except for gestational age and BMI with values of 39.9 SA respectively for channels bass, SA for 38.6 and 28.03 cesareans for low tract and 29.1 cesareans. The average values of cortisol were 403.4 nmol/L \pm 260.90 for bass tracks and $117.1 \pm 63.90$ for caesarean sections. The difference is statistically significant with a $p<\mathbf{0 . 0 0 0 0 1}$. The results of the second part showed that there are significant differences by comparing post-vaccination pain scores as well after BCG to 15 days of life, after Infanrix HIB polio at two months of life. $p$ is respectively 0.0016 at 15 days of life and 0.0001 at two months. In conclusion, newborns' extracts assisted vagina to live such stress at birth; their immediate response to stress is much higher than those born by high way before the beginning of work, and their pain response is more intense in 15 days and two months of life.
\end{abstract}

\section{Keywords}

Answer, Stress, Newborn, Vaginally, Assisted

\footnotetext{
${ }^{*}$ Corresponding author.
}

How to cite this paper: Radouani, M. A., Chahid, N., Kharbach, A., Mrabet, M., Hassani, A., Aguenaou, H., \& Barkat, A. (2015). Influence of Mode of Delivery on the Stress Response in Infants. Psychology, 6, 1861-1872. 


\section{Introduction}

Stress can be defined as state mentally or emotionally disruptive or upsetting, occurring in response to external influences. Stress experienced by a woman during her pregnancy can affect her physical and emotional functioning (Canouï, 1998; Laborit, 1988).

Maternity stressing the newborn is a daily reality. The newborn term is particularly confronted with it, whether or not obstetric risk factors. Birth, obstetric complications and the care of the newborn at birth, are all actions that can induce stress and pain. Among large hospitals' perinatal specialists, only a small number chose to establish a treatment protocol of newborns with clinical signs or risk factors at birth room (Bensabat, 1980, Boudarene, 1997).

Therefore, many hospitals do not have the clinical tools and materials needed for diagnosis and simple treatment of the first painful signs in a newborn futures in the first hours of life. Many of them rely on good clinical sense. Some prescribe painkillers or implement a different strategy associated with monitoring.

Recognizing this, we wondered about the reality of neonatal stress in the delivery room, and the response of the newborn to stress in the short and medium term. We chose to conduct a comparative study evaluating neonatal stress in two newborn populations, one vaginally after instrumentalizing and the other by caesarean section in the CHIS maternity Rabat, Morocco.

We have cleared the following question: Are preventive measures analgesics in neonates required for any delivery instrumentalized?

\section{Methodology}

This is a prospective study conducted at the Maternity Souissi Rabat and Neonatology at the National Reference Center and Nutrition of children Rabat. We included 25 newborns born vaginally assisted and 25 newborns extracted by cesarean. Newborns of both groups are reviewed for BCG vaccination at the age od 15 days and at the age of two months for INFANRIX Polio Hib vaccine.

Vaccination and evaluation of the pain response are carried out by two operators, but the same each time.

- Inclusion criteria: Women aged 18 - 45 years, free from any disease, pregnant and full term pregnancy.

- Exclusion criteria: Newborns must be free of any pathology.

\subsection{Design of Study}

- The conduct of the study

\section{$>$ For women}

They are recruited in the delivery room. The protocol has been clearly explained and after informed consent, they are included in the study if their delivery required the use of a suction cup. The same approach was followed for scheduled cesareans. A fact sheet is filled for every woman recruited. It includes data on the parturient, her gravidity and parity, blood pressure, weight and size and mode of delivery.

At delivery, whatever the mode:

1) Five minutes after the clamping is carried out on a sample dry flask in umbilical artery for determination of cortisol. This levy is sent within 12 hours to the laboratory where it is centrifuged at $3000 \mathrm{G}$ at $4^{\circ} \mathrm{C}$, and then aliquoted and frozen at $-80^{\circ} \mathrm{C}$ until assayed.

2) The placenta is weighed using an electronic balance accuracy.

3) Anthropometric measurements of the newborn were taken: weight, height and head circumference. The weight is determined by a balance Newborn (Seca) to $10 \mathrm{~g}$ close. All newborns are weighed naked as they complete their adaptation and review performed. The size is measured at $0.1 \mathrm{~cm}$ near by a pediatric fathom. The measurement head circumference is effected by means of a tape at $0.1 \mathrm{~cm} .4$-Sex noted.

\section{For newborn}

At admission in consultation with day 15 and two months of life infants are examined, they present their health card. The operators are not seeking to know which of the two groups before the child belongs to vaccinate and evaluate it. The children did not receive any analgesic means and a minimum interval of 30 minutes is allowed between the last feeding and vaccination act. The child placed on her mother's lap. The vaccination site was:

- The Latero-external part of the arm at the upper third in strict intradermal to BCG 
- The Latero-lateral aspect of the thigh to the INFANRIX Polio Hib.

DAN grids are met after watching the facial expression, vocal expression and body movements of the newborn for 2 minutes before vaccination at the time of vaccination, and 3 minutes after removing the needle. The data is recorded on a listing vaccination.

- Pain Assessment Method

The choice was for the use of acute pain rating scale newborn "Scale DAN". The primary endpoint was evaluation among newborns of pain induced by the vaccine act using the DAN scale. This is a behavioral grid, 3 items with 4 - 5 possible responses for each of them. Facial motor skills, body movement but also the analysis of the cry are analyzed signs.

- Cortisol Assay method

The assay was done by radio-immunoassay. Direct measurement of cortisol in serum. Reconstitution standard with $0.5 \mathrm{ml}$ distilled water. Cap the bottle and mix gently to ensure complete dissolution of the lyophilized product.

- Experimental Protocol

All reagents must be brought to room temperature $\left(18^{\circ} \mathrm{C}-25^{\circ} \mathrm{C}\right)$ at least 30 minutes before use. The distribution of the reactants in the coated tubes must be at room temperature. The assay requires the following tubes: Total tubes $(\mathrm{T})$, the standard tubes, control tubes and the samples to be assayed. The determination of cortisol itself comprises:

- Distribute 20 mu.l of standard or samples into the corresponding tubes.

- Add 500 mu.l of the 125 I tracer in cortisol all tubes including totals tubes.

- Vortex

- Cover the tubes with parafilm

- Incubate for 2 hours at $37^{\circ} \mathrm{C}$.

- Remove the solution by inversion and tapping the tubes manually (except totals)

- Add $1 \mathrm{ml}$ of distilled water (except the tubes T)

- Eliminate The solution by inversion and tapping the tubes back on paper towels and leave 2-3 minutes on paper towels (except totals)

- Measure the radioactivity bound to the tubes coated using a gamma scintillation counter set to the measurement of iodine-125.

\subsection{The Statistical Study}

All data collected through the farm returns were entered into Excel and then exported for analysis using SPSS 9.0 software. The analysis was conducted at the Laboratory of Biostatistics and Clinical Research and Epidemiology (LBRCE) of the Faculty of Medicine and Pharmacy of Rabat. Pars also to obtain $80 \%$ power and $5 \%$ significance in the detection of a 2-point difference in the scale of DAN, 25 newborns should be introduced in each group. Thus even newborns 7 excluded cortisol assays (including the two who experienced respiratory distress at birth and five hemolyzed serum) were included in this second part of the study.

The statistical analysis comprised two stages, he acted in the descriptive analysis and the univariate analysis that aims to determine whether there is a statistical relationship between two variables and that by calculating the coefficient correlation between them is the coefficient $(p)$. We say that two variables were significantly associated if the calculated correlation coefficient is less than $0.05(p<0.05)$. Qualitative data were compared with chi-square test of Pearson.

\section{Results}

\subsection{Descriptive Study}

1) Group newborns born by cesarean

25 women were recruited in this group. All caesarean sections are performed under spinal anesthesia and with the same anesthetic protocol. No complications were recorded in mothers and no women had received corticosteroids.

Data on maternal characteristics and newborns included in the Tables 1-3. Regarding the determination of 
cortisol it has interested that 18 of 25 samples, two sera were excluded because the infants had developed respiratory distress in connection with a late resorption and 5 were hemolyzed sera.

Table 4 specifies the main indications of cesarean sections.

2) Group newborns born vaginally

The 25 women recruited in this group had no analgesia. Working time was less than 12 in any case. Extraction necessitated the use of a suction cup in every case. Childbirth gave birth to 25 healthy newborns. No serum was haemolysed in this group. Cortisol assay was performed on all newborns. Data on maternal characteristics and newborns included in the Tables 5-7.

Table 1. Maternal data.

\begin{tabular}{|c|c|c|c|c|}
\hline Variable & Average & SD & Minimum & Maximum \\
\hline Maternal weight (Kg) & 72.7 & 19.2 & 50.0 & 117.0 \\
\hline Maternal size $(\mathrm{cm})$ & 157.8 & 5.4 & 148.0 & 167.0 \\
\hline Maternal BMI & 29.1 & 6.9 & 20.8 & 44.5 \\
\hline Gravidity & 2.9 & 2.7 & 1.0 & 11.0 \\
\hline Parity & 2.4 & 1.5 & 1.0 & 7.0 \\
\hline Maternal age (years) & 28.9 & 11.8 & 17.0 & 41.0 \\
\hline
\end{tabular}

Table 2. Neonatal data.

\begin{tabular}{ccccc}
\hline Variable & Average & Minimum & Maximum & SD \\
\hline Gestational age (WA) & 38.6 & 37.0 & 41.0 & 1.03 \\
Birth weight (g) & 3504.4 & 2700.0 & 4800.0 & 660.60 \\
Size (cm) & 50.0 & 48.0 & 53.0 & 19.30 \\
Head circumference (cm) & 35.8 & 33.0 & 39.0 & 1.77 \\
Placenta weight (g) & 611.0 & 450.0 & 970.0 & 140.00 \\
Cortisol rate (blood corde) & 117.1 & 40.9 & 244.8 & 63.90 \\
\hline
\end{tabular}

Table 3. Sex repartition.

\begin{tabular}{ccc}
\hline Sex & Frequency & Percentage \\
\hline Female & 6 & 33.3 \\
Male & 12 & 66.7 \\
\hline
\end{tabular}

Table 4. Indications of the caesarians.

\begin{tabular}{ccc}
\hline & Frequencies & Percentages \\
\hline Contracted pelvis & 9 & 50.0 \\
Scared uterus & 4 & 22.0 \\
Asymmetry of pelvis & 1 & 6.0 \\
Macrosomia & 4 & 22.0
\end{tabular}


Table 5. Maternal data.

\begin{tabular}{ccccc}
\hline Variable & Average & SD & Minimum & Maximum \\
\hline Maternal weight (Kg) & 71.40 & 11.05 & 53.00 & 100.00 \\
Maternal size (cm) & 159.80 & 27.30 & 150.00 & 170.00 \\
Maternal BMI & 28.03 & 4.49 & 19.70 & 36.70 \\
Gravidity & 2.04 & 0.93 & 1.00 & 1.00 \\
Parity & 2.00 & 0.90 & 17.00 & 4.00 \\
Age (years) & 27.60 & 5.90 & 40.00 \\
\hline
\end{tabular}

Table 6. Neonatal data.

\begin{tabular}{ccccc}
\hline Variable & Average & SD & Minimum & Maximum \\
\hline Gestational age (SA) & 39.90 & 0.80 & 38.00 & 41.00 \\
Birth weight (g) & 3411.00 & 407.00 & 2100.00 & 4300.00 \\
Size (cm) & 49.20 & 2.20 & 44.00 & 53.00 \\
Head circumference (cm) & 33.70 & 1.80 & 30.00 & 37.00 \\
Placenta weight (g) & 575.30 & 109.10 & 300.00 & 850.00 \\
Cortisol (blood corde) & 403.40 & 260.90 & 147.00 & 1227.00 \\
\hline
\end{tabular}

Table 7. Sex repartition.

\begin{tabular}{ccc}
\hline Sex & Frequency & Percentage \\
Female & 11 & 44.0 \\
Male & 14 & 56.0 \\
\hline
\end{tabular}

\subsection{Analytical Study}

The analytical study involved the comparison of two groups of newborns regarding the main variables analyzed, the search for the possible existence of a significant difference in cortisol levels analyzed the blood of the umbilical artery between the two groups, the possible influence of certain parameters on cortisol levels namely: sex, birth weight and placental weight.

- At birth

According major maternal variables analyzed Comparing the two groups studied by major variables analyzed knowing: maternal weight, maternal height, maternal BMI, parity and age of mother are shown in Table 8. It is noted that the maternal characteristics between the two groups are identical except with respect to BMI.

$\checkmark$ According to leading neonatal variables

Table 9 shows the different neonatal variables (gestational age, birth weight, height, cranial perimeter, weight of the placenta and cortisol rate) in both study groups. Comparing the characteristics of newborn shows a significant difference in gestational age and average cortisol levels were significantly higher among those born with vaginally delivery.

$\checkmark$ Changes in cortisol levels within each group

As regards the possible influence of certain parameters on cortisol levels, we became interested in finding the existence within each group of a difference in cortisol levels based on gender, weight birth, gestational age and placental weight. Sex Table 10 shows that regardless of the type of delivery there are no significant differences in cortisol levels in both sexes.

Possible influence of birth weight, gestational age and placental weight on cortisol levels analysis of Table 11 shows that just no correlation was found between cortisol levels and birth weight, gestational age and placental weight. 
Table 8. Comparing the two groups on key maternal variables analyzed.

\begin{tabular}{cccc}
\hline Variable & Average Vaginal delivery & Average Cesarian & $P$ \\
\hline Maternal weight (Kg) & 71.40 & 72.7 & 0.35 \\
Maternal size (cm) & 159.80 & 157.8 & 0.56 \\
Maternal BMI & 28.03 & 29.1 & $\underline{0.0001}$ \\
Gravidity & 2.04 & 2.9 & 0.71 \\
Parity & 2.00 & 2.4 & 0.58 \\
Age (years) & 27.60 & 28.9 & 0.27 \\
\hline
\end{tabular}

Table 9. Different neonatal variables.

\begin{tabular}{cccc}
\hline Variable & Average Vaginal delivery & Average Ceasarian & $\boldsymbol{p}$ \\
\hline Gestational age (WA) & 38.9 & 38.6 & 0.06 \\
Birth weight (g) & 3411.0 & 3504.4 & 0.558 \\
Size (cm) & 49.2 & 50.0 & 0.191 \\
Head circumference (cm) & 33.7 & 35.8 & 0.166 \\
Placenta weight(g) & 575.3 & 611.0 & 0.278 \\
Cortisol (corde blood) (nmol/l) & 403.4 & 117.1 & $\leq 0.00001$ \\
\hline
\end{tabular}

Table 10. Cortisol rates according mode of delivery.

\begin{tabular}{cccc}
\hline Mean rates of cortisol $(\mathbf{n m o l} / \mathbf{l})$ & Female & Male & $p$ \\
\hline Vaginal delivery & 356.2 & 440.6 & 0.7 \\
Cesarian & 126.6 & 107.7 & 0.6 \\
\hline
\end{tabular}

Table 11. Correlations between cortisol levels and birth weight, GA and placental weight.

\begin{tabular}{ccc}
\hline Variable & R & IC $_{95 \%}$ \\
\hline Birth weight & -0.15 & $-0.56-0.57$ \\
Gestationnel age & 0.22 & $0.25-0.34$ \\
Placenta weight & 0.04 & $-0.34-0.38$ \\
\hline
\end{tabular}

- Influence of mode of delivery on the painful response to vaccination and 15 days of life

1) Status of newborns before vaccination

The status of newborns before vaccination was considered. A newborn who cries is excluded temporarily until he calms down. The presence of tears not to establish the score of DAN since it takes into account the vocal expression. The distribution of newborns who were awake or asleep without crying in the 2 groups before the vaccine act is shown in Figure 1. The median of the DAN scores collected prior to vaccination, are all equal to 0 , meaning no newborn has presented a pain before (Table 12).

- Pain assessment by DAN scores for BCG

Pain scores collected by the DAN scale during the realization of the BCG, are shown in Figure 2.

It is clearly seen that the vaccine act is very painful in the vaginal group instrumented. In fact, the median is at 8 and the lower limit of the interquartile range to 6 . This means that $75 \%$ of infants showed higher pain or equal to 6 on a scale of 0 to 10 . In the group born by cesarean medians are equal to 5 . The upper limit of the interquartile range was 8 , which means that only $20 \%$ of infants experienced a higher pain to 8 on a scale from 0 to 10 . 


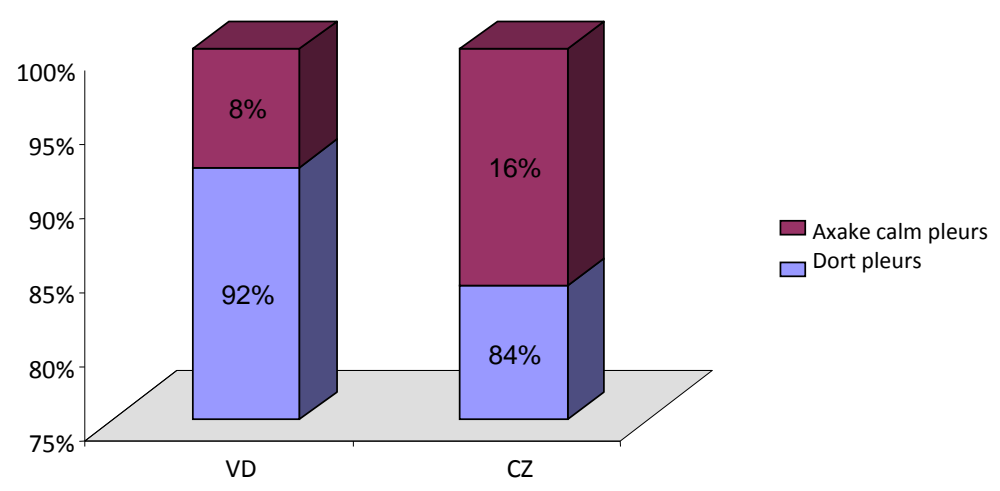

Figure 1. The status of newborns before vaccination in the two groups G1: newborns from VD BCG. G2: n-born from cesarean BCG.

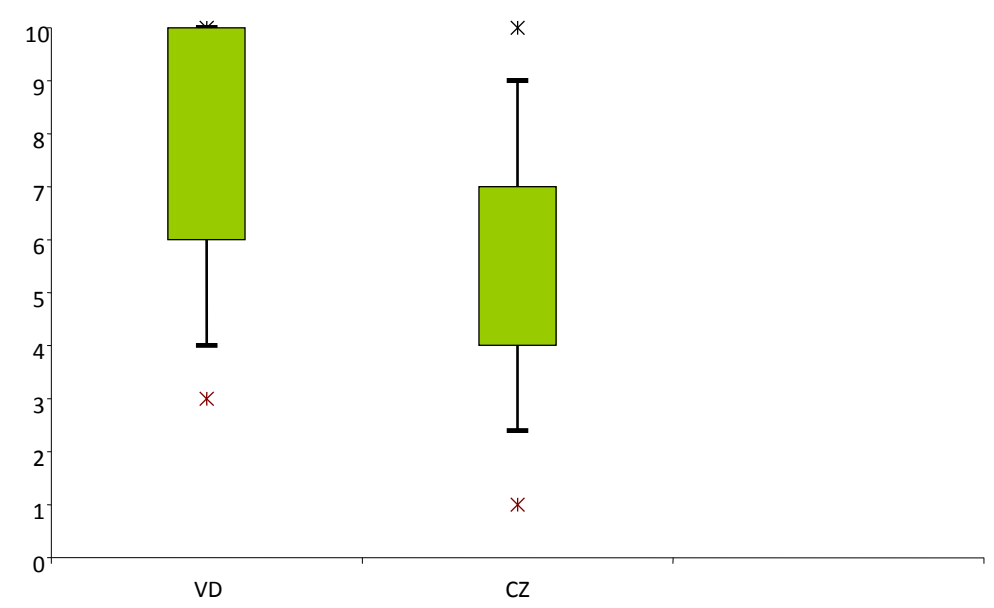

Figure 2. DAN scores comparison during the BCG.

Table 12. Averages and medians of DAN scores in the two groups during production of the BCG.

\begin{tabular}{cccccc} 
& Average & IC $_{95 \%}$ & Median & Interquartile rangs & Minimum - Maximum \\
\hline Vaginal delivery & 7.52 & {$[6.56-8.48]$} & 8 & $6-10$ & $3-10$ \\
Ceasarian & 5.24 & {$[4.19-\mathbf{6 . 2 9}]$} & $\mathbf{5}$ & $\mathbf{4 - 7}$ & $\mathbf{1}-\mathbf{1 0}$ \\
\hline
\end{tabular}

The horizontal bars represent medians, boxes indicate the interquartile ranges and the vertical lines deciles, are also represented extreme values as stars.

2) Pain assessment by DAN scores after BCG vaccination

Table 13 shows medians, interquartile ranges and minimum-maximum intervals DAN scores after completion of BCG, in the 2 groups.

For the VD group, the median is 0 and the upper limit of the interquartile range to 4 . This means that $25 \%$ of infants showed greater pain than or equal to 4 on a scale of 0 - 10 after BCG. Pain scores obtained in infants born by cesarean section were given equal medians 0 so over $75 \%$ had no pain score.

3) Status of newborns after vaccination

The condition of hatchlings after vaccination is compared between the two groups. On Figure 3, we see that $40 \%$ of newborns are still crying after completion of BCG in the VB group. In the group of caesarean approximately $70 \%$ of newborns were calm after BCG.

Comparing DAN scores between the two groups

During the vaccination act comparing DAN scores between the two groups showed a significant difference in pain scores obtained at the time to the vaccine with a $p=0.016$. 


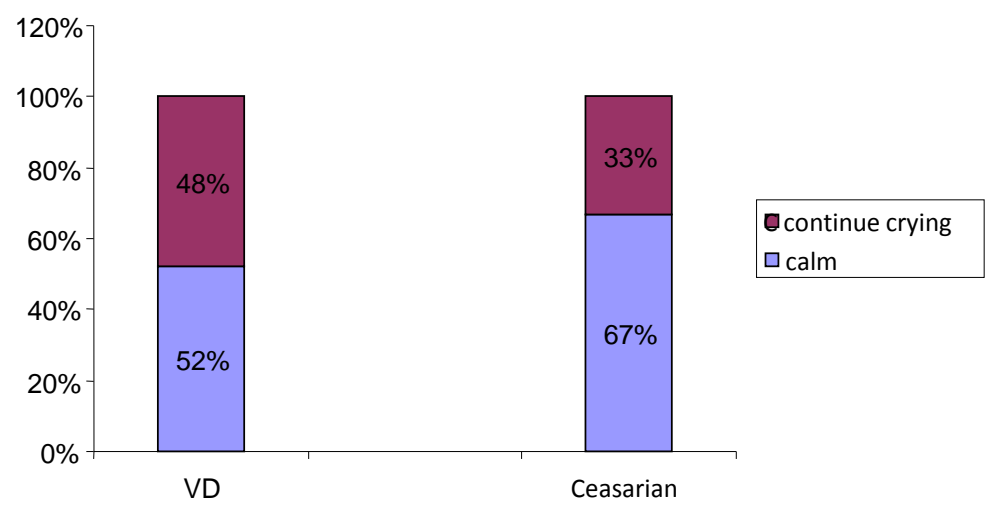

Figure 3. The status of newborns after vaccination in both groups.

Table 13. Pain assessment with the DAN scale after completion of BCG in two groups.

\begin{tabular}{cccccc} 
& Median & Minima & $\mathbf{1}^{\text {st }}$ quartile & $3^{\text {rd }}$ quartile & Maxima \\
\hline Vaginal delivery & 0 & 0 & 0 & 4 & 8 \\
Ceasarian & 0 & 0 & 0 & 0 & 6 \\
\hline
\end{tabular}

In Table 14 is shown the $p$ value in the comparison groups are given medians and interquartile ranges DAN scores.

- Influence of mode of delivery on the pain response after vaccination with two months of age

4) Status of newborns before vaccination

The distribution of newborns who were awake or asleep without crying in the 2 groups before the vaccine act is shown in Figure 4. Medians DAN scores collected prior to vaccination, are all equal to 0, meaning that no newborn showed pain before.

$>\quad$ Pain assessment by the DAN scores during INFANTIX vaccination Polio Hib (2 months)

Table 15 shows the means and medians DAN scores obtained during the realization of the vaccination in both groups.

It is clearly seen that the vaccine act is painful in the vaginal group instrumented (Figure 5). In fact, the median is 6 and the upper limit of the interquartile range to 8.

It means that $20 \%$ of newborns presented a pain greater than or equal to 8 on a scale of 0 to 10 . In the cesarean group, the median score was 3, with an upper limit of the interval interquartile to 4 , which means that $75 \%$ of newborns have presented a pain score less than 4 on a scale of 0 to 10 (Table 16).

For the vaginal group, the median is 0 and the upper limit of the interquartile range to 4 . This means that $25 \%$ of infants showed greater pain than or equal to 4 on a scale from 0 - 10 after BCG. Pain scores obtained in infants born by cesarean section gave medians equal to 0 . They were quiet 2 minutes after the vaccine act.

Comparing DAN scores between the two groups during the vaccination act Comparing DAN scores between the two groups showed a significant difference in pain scores obtained at the time to the vaccine with a $p=$ 0.0001 (in Table 17).

\section{Discussion}

Adaptation to extra-uterine life has tremendous stress that can be described as "good" stress since it allows to perform the transition. But a newborn infant subjected to aggression such an instrumental extraction under stress can become biologically "bad" when it exceeds the level of own adaptation of the individual. Nevertheless, we can't deny that there is a large inter-individual variability regarding how one receives and interprets events, it influences the body’s response (Trevor, 2005; Kristina, 2006; James, 2003; Hazard, 1990; Cambier, 1995).

The finding that neonates from an instrumented delivery are difficult consolable led to several studies on the effects of mode of delivery on the stress response at birth and a few months later. This memory is placed in this context. 


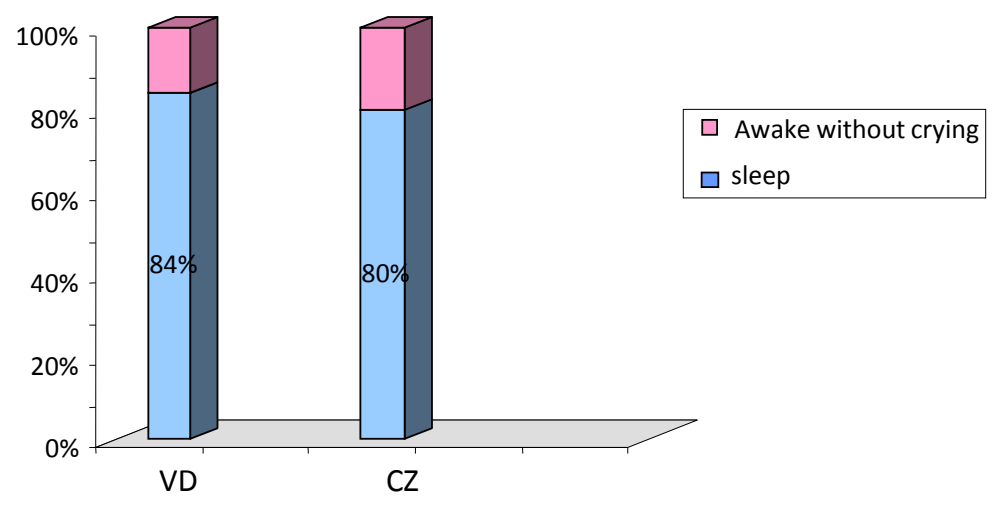

Figure 4. The status of newborns before vaccination in the two groups.

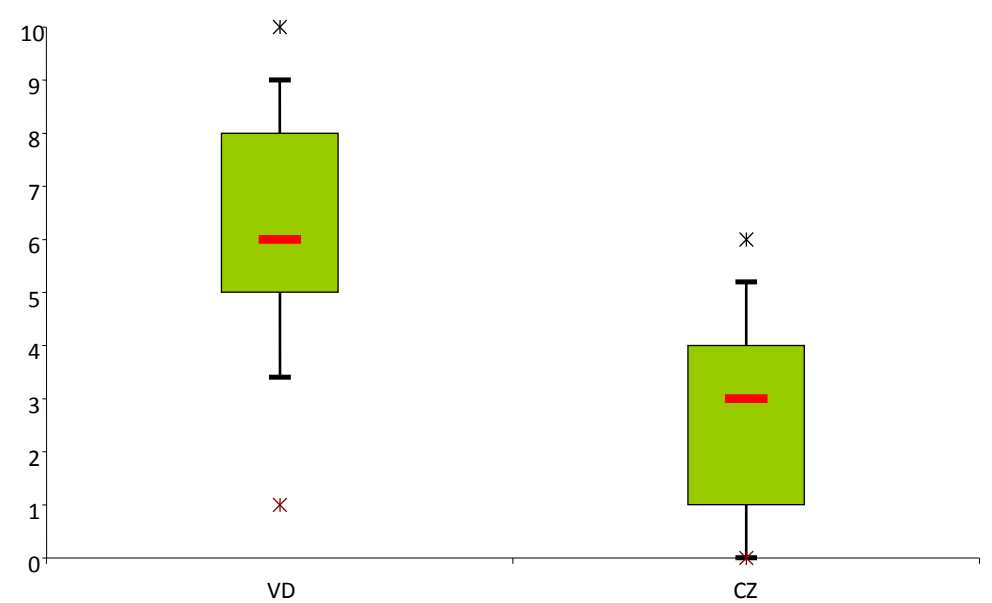

Figure 5. Pain scores collected by the DAN scale during the realization of the vaccine are shown in.

Table 14. Comparison of the DAN scores between the two groups during the vaccination act.

\begin{tabular}{cccc}
\hline & Median & Interquartile rangs & $P$ \\
\hline Vaginal delivery & 8 & $(6-10)$ & $\underline{0.016}$ \\
Ceasarian & 5 & $(4-7)$ & \\
\hline
\end{tabular}

Table 15. Averages and medians of DAN scores in the two groups during the implementation of INFANTIX Polio Hib vaccine.

\begin{tabular}{cccccc}
\hline & Average & IC $95 \%$ & Median & Interquartile rangs & Max - Min \\
\hline Vaginal delivery & 6.16 & {$[5.23-7.09]$} & 6 & $5-8$ & $1-10$ \\
Cesarian & 2.56 & {$[1.77-3.35]$} & 3 & $1-4$ & $0-6$ \\
\hline
\end{tabular}

Table 16. Means and medians of the DAN scores in the two groups after completion of INFANTIX Polio Hib vaccine.

\begin{tabular}{ccccc} 
& Median & Minima & $\mathbf{1}^{\text {st }}$ quartile & $3^{\text {rd }}$ quartile \\
\hline Vaginal delivery & 0 & 0 & 0 & 4 \\
Ceasarian & 0 & 0 & 0 & 0 \\
\hline
\end{tabular}


Table 17. Comparison of the DAN scores between the two groups during the vaccination act.

\begin{tabular}{cccc} 
& Median & Interquartile range & $p$ \\
\hline Vaginal delivery & 6 & $(5-8)$ & $\underline{0.0001}$ \\
Ceasarian & 3 & $(1-4)$ & \\
\hline
\end{tabular}

Thus, we became interested in the analysis of the stress response of two newborn groups: the first includes 25 infants born by assisted vaginal delivery and 25 other second born by elective caesarean section. Stress responses have included the neonatal period the analysis of cortisol levels in the cord blood, and at the age of 15 days and 2 months evaluating the intensity of the pain response to vaccine act. The hypothesis being tested was that infants extracts assisted vaginal lived such stress at birth than their immediate response to stress would be significantly higher than those born by high way before the beginning of work. This stress involving overstimulation HPA axis would lead to lasting changes in the response of it at least until the age of two months which will be reviewed newborns for a second vaccination.

Our assumptions were verified. Since the cortisol levels in cord blood and pain scores after vaccination at the age of two weeks and two months were significantly higher among infants born by assisted vaginal delivery. The two groups were comparable. A significant differences were noted regarding gestational age, but this difference is unavoidable as scheduled cesarean are usually performed between 37 and 38 WA. This outcome is not a problem for this study since all newborns are futures and since cortisol levels are significantly higher than in preterm births (Mignée, 1991).

The second significant difference between the two groups interested maternal BMI. Constat which is also not a problem since it was interested in cortisol and not to leptin and where the sample is carried in the blood of the umbilical artery reflects fetal secretion. The evaluation of the stress response turned to birth, determination of cortisol in the blood of the umbilical artery. Indeed, the choice of the umbilical artery blood is motivated by the fact that the hormones contained therein are largely of fetal origin. Besides the great criticism that made several similar work was to have built the conclusions referring to the results of umbilical venous blood (Olivier, 1997; Paulmyer-Lacroix, 1995; Mills, 1985; James, 1997; Gitau, 2007).

It contains hormones largely via maternal placental filter. Nevertheless, even on venous blood findings were the same. Anyway several studies have shown positive correlations between cortisol levels in the umbilical venous and arterial blood.

Another criticism is often made in articles having treated the same subject, it not precise the type of anesthesia used and the type of anesthetic. The drugs had interference on the stress response. All patients of our study were had caesareans under spinal anesthesia and with the same anesthetic protocol. For the vaginally delivery no analgesia was used (Stephen, 2007; Owen, 2007; Bell, 2007; Vogl, 2006; Mears et al., 2004; Gitau, 2007).

On other side, there is now no doubt that the delivery pains are felt to be an intensive experience for the newborn whose pain perception functions are already sufficiently mature early in the intrauterine life. Furthermore dystocic and instrumented delivery is a superimposed test that increases pain perception in fetuses. Fact which results in an increase in cortisol levels, but also catecholamines and beta endorphins in cord blood.

Nevertheless, our study can not claim these demonstrate whether high cortisol levels in case of delivery by instrumental vaginal delivery are related to obstetric reasons or using the suction cup. It should have been a third group have made delivery without instrumentation. Indeed, in case of a normal delivery the compression exerted on the fetal skull is between 8 and $15 \mathrm{~kg}$. One of these passes 15 to $20 \mathrm{~kg}$ in case of using cup and 16 to $23 \mathrm{~kg}$ in the case of extraction forceps. It explains why we chose to compare about newborns suffering the same stress mode.

The question is: what stress and pain caused by an instrumented delivery they have derogatory effects on the response to short and medium-term stress?

While the stress of a normal delivery, the cascade of adaptive responses that involves, allows the newborn to adapt to the extra-uterine life. It helps eliminate fluid and intra alveolar to preserve blood flow to vital organs during hypoxic times. The medical findings have also clearly shown the high frequency of respiratory distress and transient tachypnea of newborn infants born by elective caesarean section (two cases in our small series). But the perceived stress after an assisted birth is so important that it is likely to be associated with a perception of more intense pain in a normal delivery. 
Data from animal studies have shown that perinatal stress can have lasting effects on the HPA axis. And a significant pain during the first days of life can increase the pain response. These findings were verified by looking at newborns with painful situations experienced either acute or chronic in the first days of life. The typical example of acute pain is represented by a circumcision. Indeed, it was believed that circumcision without analgesia in the neonatal period was a short quickly forgotten unpleasant experience. Now studies are being interested in assessing the response to the post vaccination pain weeks after circumcision showed significant differences between girls and boys and between circumcised and uncircumcised boys (Taddio et al., 1998).

Other studies have evaluated the effect of mode of delivery on the response to a vaccine act at the age of two months using as judgment criteria cortisol levels in saliva after vaccination and evaluating the response the vaccine act by behavioral scales. They have clearly demonstrated that the response to the pain of children born by Caesarean section, evaluated by cortisol levels and behavioral scales are lower than those born vaginally instrumentation. The evaluation of the stress response after vaccination act in our study was based on the use of a sharp pain assessment score to know the score of DAN. The inter-observer agreement is good. The majority of similar studies were based on a single endpoint that is the quality of the cry. The grid we used takes into account in addition to the analysis of the cry, the body movements. Its internal consistency assessed by Cronbach is excellent with a coefficient of 0.88 (Debillon, 1998). There are strong arguments for its construct validity, by comparing scores between a group of children and a painful painless group (significant difference $p<0.0001$ ) (Carbajal, 1997). Although this grid has been the subject of a validation of its quality metric with the demonstration of good sensitivity intra- and interindividual and scores were made by the same operator who does not know which of the two groups located the newborn. We would have liked to cortisol assay and concomitantly also in saliva. However, we were limited by the means. By the results of the grids used, it has been shown that infants born by low voice instrumented have significantly higher pain scores than those born by caesarean section as well in 15 days to two months. By cons, we can not compare the evolution of the response to pain in the same group as the two vaccination techniques are different.

These early events will they affect brain function? Can the events of our early childhood program our brain?

The development of our nerve cells is extremely sensitive to various molecules such as growth factors, gene transcription factors and hormones, including steroid hormones, which can affect cell renewal, migration and formation of nerve networks. Animal models have been used to demonstrate these phenomena, in particular the consequences of manipulating the prenatal environment, such as prenatal stress, in which the pregnant mother is subjected to an unscheduled stressor as intense light or noise. The potential candidates were naturally steroids released by the adrenal gland during stress these episodes. Although there is a barrier corticosteroids to cross the placenta (the latter producing an enzyme which destroys these hormones), it can be broken, making the fetus directly exposed to these steroids. Thus the high-dose administration of a synthetic steroid or an inhibitor of this enzyme produces the same effects as prenatal stress with a decrease in the weight, a pressure and high glucose levels in the blood. Strong corticosteroids rate result after some time in a hyperactivity of the HPA axis, or axis of the stress which results in an increase in the expression in the brain of the CRH gene which in its turn increases the release of steroids (Gitau, 2001). Specific tests have shown that small whose mothers received high doses of these steroids are more anxious. Their cognitive ability is also affected. Small thus have more trouble learning in spatial memory tests.

\section{Conclusion}

If we have a rich literature on the effects of early psychological trauma on the human brain development, as well as the long term effects of neonatal pain experiences, few data are available regarding the long term consequences of instrumented deliveries. Indeed, it is difficult to reproduce the same conditions in animals. Nevertheless, the cohort of children constitution for different delivery modalities and their long-term clinical follow-up as well, psychological and behavioral will gain valuable data. This work is not easy, however, given the large "environment" component to be taken into account. The finding is therefore, there is an exaggerated response to stress in case of vaginal instrumented and a more intense pain response at 15 days and two months. These differences may be explained with reference to animal experiments to changes in the stress response of the HPA axis.

\section{Conflict of Interests}

None. 


\section{References}

Bell, A. (2007) Don't We Want Aroused and Alert Babies at Birth? BJOG: An International Journal of Obstetrics and Gynaecology, 114, 120-121. http://dx.doi.org/10.1111/j.1471-0528.2006.01157.X

Bensabat, S. (1980). Le stress, De grands spécialistes répondent, pp 350, Ed. Hachette.

Boudarene, M. (1997). Qu'est-ce que le stress? Rev. Médicale. Liège, Belgique, 52, 541-549.

Cambier, J. (1995). Manuel de Neurologie (599 pages). Ed. Masson, 8ème édition.

Canouï, P. (1998). Le syndrome d'épuisement professionnel des soignants. Ed. Masson: 211 p.

Carbajal, R. (1997) M. DAN: Une échelle comportementale d'évaluation de la douleur aiguë du nouveau né. Archives de pédiatrie, 4, 623-628.

Debillon, T. (1998). Les grilles d’évaluation de la douleur chez le nouveau né: Revue de la littérature. Douleur et analgésie, 4, 167-172.

Gitau, R. (2007). Cord Cortisol Levels as an Indicator of the Fetal Stress Response to Assisted Vaginal Delivery. Eur J Obstet Gynaecol, 39, 1-4.

Hazard, J. (1990). Manuel d’Endocrinologie (560 pages). Ed. Masson, 3ème édition

James, P. (1997). Neurocircuitry of Stress: Central Control of the Hypothalamo-Pituitary-Adrenocortical Axis. Trends in Neurosciences, 20, 78-84.

James, P. (2003). Central Mechanisms of Stress Integration: Hierarchical Circuitry Controlling Hypothalamo-PituitaryAdrenocortical Responsiveness. Frontiers in Neuroendocrinology, 24, 151-180.

Kristina, A. (2006). Hippocampal Neuroplasticity Induced by Early-Life Stress: Functional and Molecular Aspects. Frontiers in Neuroendocrinology, 27, 180-192.

Laborit, H. (1988). Encyclopaedia Universalis, pp. 271-272, Corpus 17, Soutine Tirso.

Mears, K., McAuliffe, F., Grimes, H., \& Morrison, J. J. (2004). Fetal Cortisol in Relation to Labour, Intrapartum Events and Mode of Delivery. Journal of Obstetrics and Gynaecology, 24, 129-132. http://dx.doi.org/10.1080/01443610410001645389

Mignée, C. (1991). Le stress, Biologie et Physiologie. Archives de Maladies Professionnelles, 52, 255-257.

Mills, F. J. (1985). The Endocrinology of Stress. Aviation, Space, and Environmental Medicine, 56, 642-650.

Olivier, C. (1997). Arginine-vasopressine et fonction corticotrope lors du stress. La Presse Médicale, 26, 1635-1641.

Owen, D. (2007). Prenatal Glucocorticoid Exposure Alters Hypothalamic-Pituitary-Adrenal Function in Juvenile Guinea Pigs. Journal of Neuroendocrinology, 19, 172-180. http://dx.doi.org/10.1111/j.1365-2826.2006.01517.x

Paulmyer-Lacroix, O. (1995). Régulation de la fonction corticotrope dans les situations de stress. Annales d'Endocrinologie, 56, 225-245.

Stephen, G. (2007). Foetal Experience: Lifelong Consequences. Journal of Neuroendocrinology, $19,73$.

Taddio, A., Katz, J., Ilersich, A. L., \& Koren, G. (1997). Effect of Neonatal Circumcision on Pain Response during Subsequent Routine Vaccination. The Lancet, 349, 599-603. http://dx.doi.org/10.1016/s0140-6736(96)10316-0

Trevor, A. (2005). Defining Stress as a Prelude to Mapping Its Neurocircuitry: No Help from Allostasis. Progress in NeuroPsychophrmacology and Biological Psychiatry, 29, 1195-1200.

Vogl, S. E. (2006). Mode of Delivery Is Associated with Maternal and Fetal Endocrine Stress Response. BJOG: An International Journal of Obstetrics and Gynaecology, 113, 441-445. http://dx.doi.org/10.1111/j.1471-0528.2006.00865.x

\section{Abbreviations}

GA: gestational age

WA: Weeks of amenorrhea

SD: standard deviation

VD: vaginal delivery

CZ: ceasarian

BCG: Bacille de Calvin et Guerin 